

\title{
Anticorps anti-pentraxine au cours des maladies auto-immunes
}

\author{
Anne-Sophie Garnier, Caroline Poli, Jean-François Subra, Alain Chevailler, \\ Pascale Jeannin, Céline Beauvillain, Jean-François Augusto
}

\section{To cite this version:}

Anne-Sophie Garnier, Caroline Poli, Jean-François Subra, Alain Chevailler, Pascale Jeannin, et al.. Anticorps anti-pentraxine au cours des maladies auto-immunes. Revue Francophone des Laboratoires, 2018. inserm-02912660

\section{HAL Id: inserm-02912660 https://www.hal.inserm.fr/inserm-02912660}

Submitted on 6 Aug 2020

HAL is a multi-disciplinary open access archive for the deposit and dissemination of scientific research documents, whether they are published or not. The documents may come from teaching and research institutions in France or abroad, or from public or private research centers.
L'archive ouverte pluridisciplinaire HAL, est destinée au dépôt et à la diffusion de documents scientifiques de niveau recherche, publiés ou non, émanant des établissements d'enseignement et de recherche français ou étrangers, des laboratoires publics ou privés. 


\title{
Anticorps anti-pentraxine au cours des maladies auto-immunes
}

\author{
Anne-Sophie Garnier ${ }^{1}$, Caroline Poli ${ }^{2,3}$, Jean-François Subra ${ }^{1,3}$, Alain Chevailler ${ }^{2,3}$, \\ Pascale Jeannin ${ }^{3}$, Céline Beauvillain ${ }^{2,3}$, Jean-François Augusto ${ }^{1,3, *}$ \\ I Service de Néphrologie-Dialyse-Transplantation, CHU d'Angers, France. \\ 2 Laboratoire d'Immunologie et d'Allergologie, $\mathrm{CHU}$ d'Angers, France. \\ 3 CRCINA, INSERM UI232, Université de Nantes, Université d’Angers, labEx IGO «Immunotherapy, Graft, Oncology », Angers, France. \\ *Auteur correspondant: jfaugusto@chu-angers.fr (JF Augusto).
}

\section{RÉSUMÉ}

Les pentraxines (PTXs) sont des récepteurs solubles innés classés dans les «pattern recognition receptors》 (PRRs). Elles sont composées de deux sous-familles définies sur une base structurelle:les PTXs courtes et les PTXs longues. Les PTXs courtes sont représentées par la protéine $C$ réactive (CRP) et le composant $P$ amyloïde (SAP). La famille des $P T X$ s longues est représentée principalement par la PTX3. Les PTXs sont impliquées dans de nombreux processus biologiques. En plus de leur fonction d'opsonine antimicrobienne, les PTXs sont aussi impliquées dans la clairance des débris cellulaires et des cellules mortes (efferocytose). Une rupture de tolérance vis-à-vis des PTXs a été mise en évidence au cours de différentes maladies auto-immunes, notamment le lupus systémique et les vascularites à ANCA. Dans cette revue de la littérature sont présentées, dans une première partie, les principales caractéristiques et fonctions biologiques des molécules de la famille des PTXs. La seconde partie comporte une synthèse des connaissances concernant les auto-anticorps dirigés contre les PTXs et leur relation avec les aspects cliniques des principales maladies auto-immunes au cours desquelles ils ont été identifiés. Les Ac anti-PTX3 font l'objet d'une présentation approfondie.

\section{Mots ClÉs \\ D auto-anticorps \\ D lupus systémique \\ pentraxines \\ D rupture de tolérance \\ - vascularite à ANCA}

\section{KEY WORDS}

- ANCA-associated vasculitis

D auto-antibodies

Dentraxins

D systemic lupus

D tolerance breakdown

(C) 2018 - Elsevier Masson SAS Tous droits réservés.

\section{Abstract}

\section{Anti-PTX antibodies in auto-immune diseases}

Pentraxins (PTXs) are soluble innate receptors belonging to the family of pattern recognition receptors (PRMs). PTXs are divided in two subfamilies based on structural characteristics: short and long PTXs. Short PTXs include C-reactive protein (CRP) and serum amyloid-P component (SAP), while long PTXs are mainly represented by PTX3. PTXs are implicated in various biological processes. Besides their anti-microbial function, PTXs are also involved in cellular debris and dying cell removal (efferocytosis).A tolerance breakdown towards PTXs has been observed in several autoimmune diseases such as systemic lupus erythematosus and ANCA-associated vasculitis. In the present review, in a first part, we summarize the main characteristics and biological functions of PTXs. In a second part, we review the knowledges on anti-PTX antibodies and their relationship with clinical features of auto-immune diseases.Anti-PTX3 antibodies are the matter of an in-depth review.

\section{Liste des abréviations}

\begin{tabular}{|c|c|}
\hline Ac & : Anticorps \\
\hline ANCA & $\begin{array}{l}\text { : anti-neutrophil cytoplasmic } \\
\text { antibody }\end{array}$ \\
\hline BPI & $\begin{array}{l}\text { : Bactericidal permeability } \\
\text { increasing protein }\end{array}$ \\
\hline CRP & : protein C-reactive \\
\hline ELISA & $\begin{array}{l}\text { : Enzyme-linked immuno- } \\
\text { sorbent assay }\end{array}$ \\
\hline EGPA & $\begin{array}{l}\text { : Granulomatose hyperéosi- } \\
\text { nophilique avec polyangéite }\end{array}$ \\
\hline GPA & $\begin{array}{l}\text { : Granulomatose avec } \\
\text { polyangéite }\end{array}$ \\
\hline
\end{tabular}
SLEDAI : Systemic Lupus Erythematosus Disease Activity Index
Small cytoplasmic : sc SPAPL: Syndrome primaire des antiphospholipides
TINU : Tubulo-interstitial nephritis with uveitis
TNFa : Tumor necrosis factor alpha
VAA : Vascularite à ANCA




\section{Dossier scientifique Immunologie}

\section{Introduction}

Les pentraxines (PTXs) sont des récepteurs solubles appartenant à la famille des Pattern Recognition Receptors (PRRs) dotées de différents rôles physiologiques. Elles opsonisent les microbes et favorisent leur capture, puis leur élimination par les cellules phagocytaires. Elles jouent également un rôle important au cours de l'efferocytose, en modulant l'élimination des cellules et débris apoptotiques par les cellules phagocytaires: physiologiquement des millions de cellules apoptotiques sont ainsi éliminées par phagocytose sans provoquer de réaction inflammatoire [I]. Différentes maladies auto-immunes (MAI), et notamment le lupus érythémateux systémique (LES), sont associées à une altération du processus d'efferocytose [2,3]. De nombreuses données, obtenues à l'aide de modèles murins ou directement chez l'homme, suggèrent qu'une altération de l'expression des PTXs est directement impliquée dans les anomalies de l'efferocytose observées au cours de différentes MAl, et dans la rupture de tolérance aux antigènes du soi [2,3]. La mise en évidence d'auto-anticorps (Ac) dirigés contre les PTXs au cours du LES, des vascularites à anticorps (Ac) anticytoplasme des polynucléaires neutrophiles (ANCA) et d'autres MAl complexifie l'étude et la compréhension du rôle des PTXs au cours de la physiopathologie de ces maladies $[4,5]$.

Dans cette revue, nous présentons dans une première partie et de façon synthétique la classification et les grandes fonctions des principales PTXs. Dans une seconde partie, nous faisons une revue de la littérature des connaissances sur les Ac anti-PTXs au cours des MAl, en développant les données concernant les Ac anti-PTX3.

\section{Les pentraxines}

Les molécules de la famille des PTXs sont caractérisées par la présence d'un domaine pentraxine très conservé de 200 acides aminés localisé dans leur partie C-terminale [6]. Les PTXs sont subdivisées en deux familles: les PTXs courtes et les PTXs longues. Cette classification est structurelle, les PTXs longues comportant un domaine $\mathrm{N}$-terminal supplémentaire, absent dans les PTXs courtes (figure I).

\section{Les pentraxines courtes}

La famille des PTXs courtes est composée de la protéine $C$ réactive (CRP) et du composant $P$ amyloïde (serum amyloid $P$ component, SAP), molécules produites à la phase aiguë de l'inflammation, et détectées dans le sérum de l'homme et de la souris, respectivement [7]. La CRP (204 acides aminés) et la SAP (205 acides aminés) sont des protéines d'environ $25 \mathrm{kDa}$ qui partagent 5 I \% d'homologie [8]. Elles sont issues de la duplication d'un gène ancestral. Les PTXs courtes sont synthétisées principalement dans le foie en réponse à des signaux pro-inflammatoires [9]. Chez l'homme, la CRP est synthétisée à la phase aiguë de l'inflammation, alors que la SAP est relativement peu produite. L'inverse est observé chez la souris.

\section{Figure 1. Structure des pentraxines courtes (CRP et SAP) et de la pentraxine-3 (PTX3).}

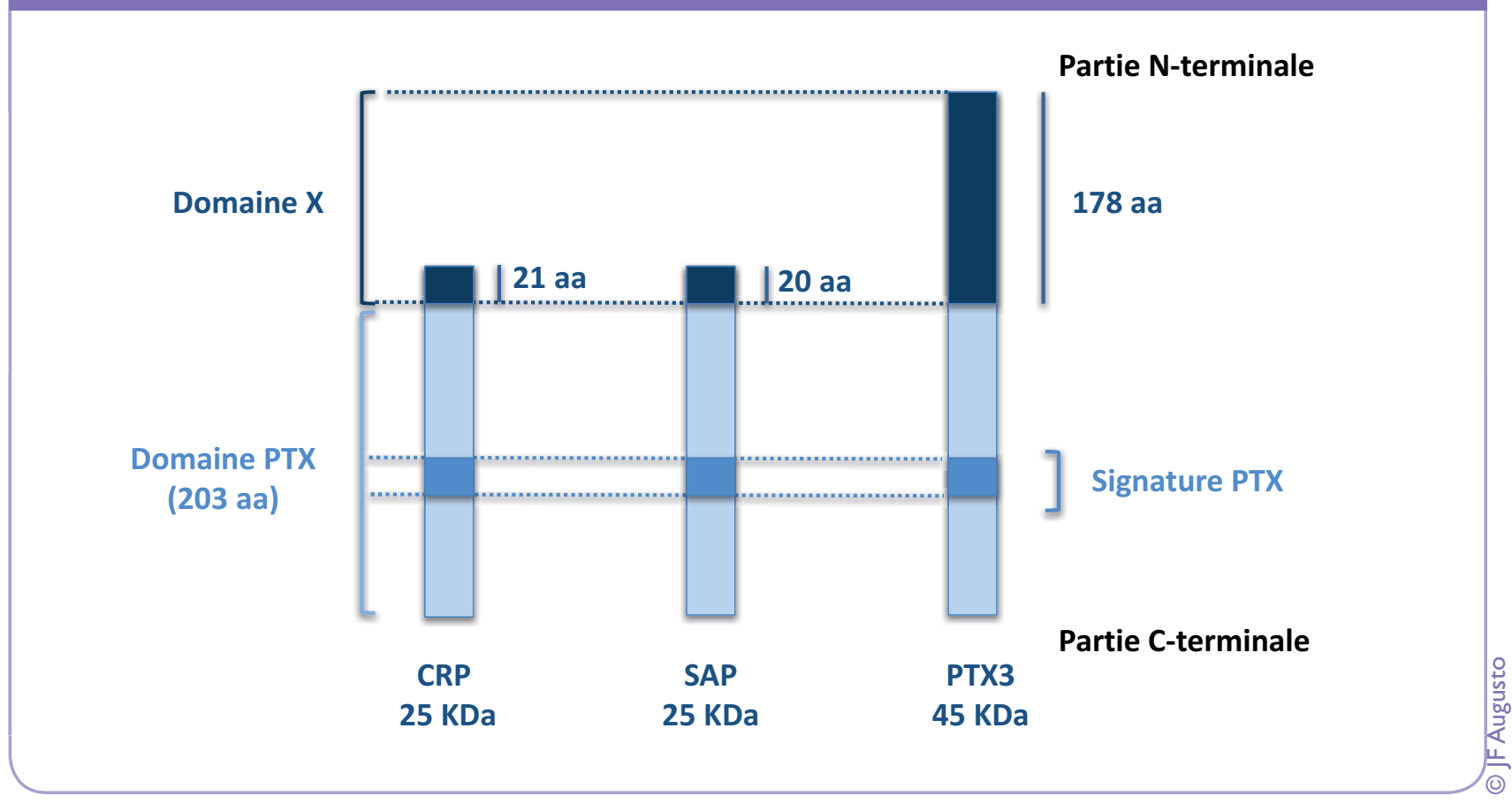




\section{La pentraxine longue, PTX3}

Pentraxine- 3 est une molécule de 45 kDa composée de $38 \mathrm{I}$ acides aminés, dont le gène est localisé sur le chromosome 3 [8]. PTX3 est très conservée, un degré d'homologie supérieur à $80 \%$ est observé entre les espèces. La partie C-terminale de PTX3 présente une forte homologie avec la partie C-terminale des PTXs courtes [8]. Cependant, PTX3 comporte une partie $\mathrm{N}$-terminale de 178 acides aminés, bien plus étendue que celle des PTXs courtes. PTX3 est organisée en octamères, les monomères étant reliés par des ponts disulfures. PTX3 est fortement sécrétée à la phase aiguë de l'inflammation, et il a été montré que sa libération survenait de façon plus précoce que celle de CRP chez l'homme. L'expression de PTX3 est induite par de nombreux stimuli. Ainsi PTX3 est synthétisée en réponse à des motifs micro-

biens, mais également des cytokines proinflammatoires (interleukine-I [IL-I], Tumor Necrosis alpha [TNF $\alpha$ ], IL-6) $[10,1 \mathrm{I}]$. Contrairement aux PTXs courtes à synthèse essentiellement hépatique, PTX3 est produite dans de nombreux tissus et par de nombreux types cellulaires, par des cellules hématopoïétiques (cellules myéloïdes) et non hématopoïétiques (fibroblastes, cellules endothéliales) $[6,8]$.

PTX3 est exprimée dans le polynucléaire neutrophile (PNN) où elle est localisée dans les granules spécifiques lactoferrine positifs [12]. Aussi, il a pu être observé que PTX3 se retrouve avec les autres protéines du PNN (PR3, MPO) au sein des expansions cytoplasmiques caractéristiques de la mort cellulaire des PNN par NETose (Neutrophil Extra-cellularTraps) [12,13].

\section{Ligands et fonctions physiologiques des pentraxines}

Les pentraxines ont de multiples fonctions, à l'interface entre immunité innée et réponse inflammatoire. Elles sont caractérisées par leur capacité à lier de nombreux ligands, certains étant communs aux deux familles de PTXs. À l'instar des PTX3, les PTXs courtes interagissent avec leur ligand de façon dépendante du calcium [8]. Les PTXs sont impliquées dans la clairance des microbes par les phagocytes, où elles agissent en facilitant leur élimination par opsonisation.Ainsi, CRP peut se lier à différents pathogènes, comme Streptococcus pneumoniae ou Haemophilus influenzae, et SAP interagit avec diverses bactéries gram négatif $[14,15]$. Le rôle anti-infectieux des PTXs courtes a pu être démontré à l'aide de modèles murins de sepsis expérimentaux à l'aide de souris déficientes en SAP ou sur-exprimant CRP [16,17]. De façon analogue aux PTXs courtes, PTX3 se lie à divers pathogènes et la perte d'expression de PTX3 dans des modèles murins de sepsis est également associée à une létalité plus importante [18]. En parallèle de ces propriétés anti-infectieuses, les PTXs sont impliquées dans la clairance des cellules mortes par les phagocytes, l'efferocytose [6]. Des anomalies de l'efferocytose, se traduisant par un défaut de clairance des débris cellulaires et des cellules apoptotiques, ont été objectivées dans nombreuses MAI [19]. Au cours de ces maladies, l'excès de matériel apoptotique, exprimant des antigènes du soi modifié, pourrait conduire à une rupture de tolérance vis-à-vis de ces mêmes antigènes et donc au développement d'une auto-immunité. Les PTXs courtes et les PTXs longues semblent avoir des fonctions différentes au cours de l'efferocytose [20,2I].Ainsi, les PTXs courtes en se liant aux cellules mourantes favorisent leur capture par les phagocytes, tandis que PTX3 semble se lier principalement aux cellules en apoptose tardive et diminuer la phagocytose de ces dernières. Les PTXs semblent jouer un rôle important dans le maintien de la tolérance immunitaire, ceci a été particulièrement étudié dans des modèles murins. Ainsi, dans un modèle murin de souris lupiques MLR/LPR, les souris prédisposées développent moins d'auto-Ac et ont un phénotype moins sévère lorsqu'elles sont supplémentées en CRP ou lorsqu'elles surexpriment CRP [22,23]. De même, des souris déficientes en SAP développent spontanément des stigmates d'auto-immunité, et des atteintes tissulaires telle qu'une atteinte rénale par dépôts d'immuns complexes [24]. Les souris déficientes en PTX3 ne semblent pas développer spontanément une auto-immunité. Toutefois, lorsque ces souris déficientes sont croisées avec des souris lupiques, une moindre capacité des souris à éliminer les débris apoptotiques et la survenue d'atteintes d'organes plus sévères (reins, poumons) sont observées [18].

Chez l'homme, il existe un faisceau d'argument témoignant que l'expression des PTXs est altérée au cours des MAI.Ainsi, au cours des poussées inflammatoires du LES, CRP augmente peu ou pas [25]. De façon analogue à CRP, nous avons pu observer des taux sériques faibles de PTX3 lors des poussées lupiques, alors que PTX 3 augmente de façon importante au cours du sepsis [II]. Ces données suggèrent que les PTXs pourraient être impliquées au cours de la physiopathologie des MAI, en altérant l'efferocytose. La détection d'auto-Ac dirigés contre les PTXs courtes et longues au cours de différentes MAI constitue un argument supplémentaire.

Dans les chapitres suivants, nous détaillons les connaissances actuelles concernant les Ac anti-PTXs. Nous développons plus particulièrement l'association de ces autoAc avec les aspects cliniques des MAI, en focalisant notre propos sur les Ac dirigés contre PTX3. 


\section{Anticorps anti-pentraxines courtes et maladies auto- immunes}

\section{Anticorps anti-CRP}

Les Ac anti-CRP ont été décrits pour la première fois en 1985 chez un patient atteint de LES [26], puis secondairement chez des patients atteints du «syndrome de l'huile frelaté» (" toxic oil syndrome»), intoxication alimentaire responsable d'une atteinte multi-systémique [27]. Les Ac anti-CRP ne reconnaissent la CRP que sous sa forme monomérique, les pentamères n'étant pas reconnus [27]. Ceci a son importance puisque les monomères de CRP résultent de la dissociation des pentamères, cette dernière survenant préférentiellement en milieu inflammatoire et acide, à la surface des cellules [28]. Il est ainsi présumé que c'est dans ces conditions que le monomère de CRP peut être modifié et devenir immunogène.

\section{Anticorps anti-CRP au cours du LES}

Les Ac anti-CRP ont été détectés principalement au cours du LES. Leur prévalence au cours du LES est de 48 à $78 \%$. Schönfeld et al ne les observent toutefois que chez moins de $10 \%$ des patients dans leur série [29]. Cette grande variabilité s'explique probablement par l'hétérogénéité des techniques de détection utilisées dans les travaux, la variabilité des valeurs seuils de positivité et aussi par l'hétérogénéité des populations de patients étudiés.

Différents travaux se sont intéressés au lien entre Ac anti-CRP et activité du LES. Dans un travail longitudinal réalisé chez 10 patients atteints de LES, Sjowall et al a pu montrer une association entre le taux d'Ac antiCRP et l'activité du LES évaluée par le SLEDAI (Systemic Lupus Erythematosus Disease Activity Index) [30]. Un «pic» de concentration était fréquemment observé avant la poussée de la maladie. Toutefois, ces données n'ont pas été confirmées dans l'étude de Shoenfeld et al [29]. II faut néanmoins préciser que dans cette dernière, outre la très faible prévalence d'Ac anti-CRP observée, il n'y avait pas de suivi longitudinal des patients.

La seule étude ayant analysé la relation entre Ac antiCRP et biomarqueurs «classiques» d'activité du LES est celle de Figueredo et al [3I]. Dans cette étude ayant porté sur 137 patients, le taux d'Ac anti-CRP était associé positivement avec les $A c$ anti-ADN double brin et les Ac anti-phospholipides, et négativement avec la concentration de C3 et de CRP. La présence concomitante d'Ac anti-CRP et d'Ac anti-phospholipides était associée à une fréquence accrue d'évènements thrombotiques et de pertes fœtales.

Les Ac anti-CRP ont fait l'objet d'une analyse plus approfondie au cours de la néphrite lupique [32,33]. La prévalence des Ac anti-CRP semble plus importante en cas d'atteinte rénale du LES, et dans ce contexte, le taux d'Ac anti-CRP est positivement associé au SLEDAl. De plus, il a pu être observé que les Ac anti-CRP diminuent et disparaissent fréquemment à la rémission de la néphrite, suggérant qu'ils pourraient être utilisés comme marqueurs d'activité de l'atteinte rénale. Un travail a montré que les Ac anti-CRP étaient associés avec la fonction rénale (créatininémie) et l'importance des lésions tubulo-interstitielles observées à la biopsie rénale [33]. Toutefois, aucune association n'a été observée avec l'importance des dépôts immuns et l'atteinte glomérulaire, plus spécifique de la néphrite lupique. Ainsi, en l'état actuel des connaissances, il apparaît que les Ac anti-CRP représentent davantage un marqueur d'activité du LES, qu'un marqueur spécifique de l'atteinte rénale. Leur association avec les $A c$ anti-C lq très prévalent au cours de la néphrite lupique n'a pas été analysée.

\section{Anticorps anti-CRP au cours des autres maladies auto-immunes}

Les Ac anti-CRP ont été détectés dans nombreuses autres pathologies auto-immunes (sclérodermie systémique, syndrome de Gougerot Sjogren, syndrome primaire des antiphospholipides [SPAPL]), le plus souvent à plus faible prévalence qu'au cours du LES.Au cours du SAPL, comme au cours du LES, la présence d'Ac anti-CRP est associée à une fréquence accrue de thromboses et de perte fotale [3I].Ainsi, les Ac anti-CRP pourraient être un marqueur intéressant pour stratifier le risque thromboembolique de ces patients. Enfin, les Ac anti-CRP ont été identifiés chez tous les patients atteints de TINU syndrome ("tubulo-interstitial nephritis with uveitis») dans une petite série de 9 patients, mais ces résultats n'ont pas fait l'objet d'une confirmation [34].

\section{Anticorps anti-SAP au cours du LES}

Seulement deux travaux ont étudié les Ac anti-SAP au cours du LES [29,35]. Leur prévalence était de $25 \%$ dans l'une et de $45 \%$ dans l'autre. Les Ac anti-SAP, de façon analogue aux Ac anti-CRP, étaient associés à l'activité de la maladie lupique. Toutefois, il n'a été observé aucune association des Ac anti-SAP avec des atteintes d’organe spécifiques.

\section{Dosage des Ac anti- pentraxine courtes: limites}

Dans toutes les études présentées, les Ac anti-PTX courtes étaient détectés par ELISA. La technique de dosage n'est pas standardisée et cela peut expliquer la grande variabilité de la prévalence des auto-Ac observée dans ces études. De plus, étant donné le niveau élevé d'homologie entre CRP et SAP, la spécificité des Ac détectés reste à préciser. 


\section{Anticorps anti-pentraxine 3 au cours des maladies auto- immunes}

Les Ac anti-PTX3 ont été décrits pour la première fois en 2009 par notre groupe au cours du LES [4], puis secondairement au cours des vascularites à ANCA [5].

\section{Anticorps anti-PTX3 au cours du LES}

Dans notre série initiale [4], nous avons observé la présence d'Ac anti-PTX3 chez $50 \%$ des patients atteints de LES (I8/36) et dans 42/I 39 (30.2\%) sérums de ces patients prélevés à des phases différentes de leur maladie. Une prévalence similaire a ensuite été observée dans une autre étude [36]. Dans notre série, nous avons observé une association entre les Ac anti-PTX3 et l'activité du LES. Les Ac anti-PTX3 étaient corrélés positivement avec les Ac anti-ADN double brin et les Ac antinucléaires, et négativement avec les fractions $\mathrm{C} 3$ et $\mathrm{C} 4$ du complément. Tous les patients avec néphrite lupique active avaient des Ac anti-PTX3 détectables au moment de la poussée. Nous avons par ailleurs observé une corrélation positive entre le taux d'Ac anti-PTX3 et la sévérité de la néphrite lupique (figure 2).Ainsi, ces données suggèrent que les Ac anti-



PTX3 pourraient être utilisés comme marqueurs d'activité du LES et possiblement de la néphrite lupique. Toutefois, dans une seconde étude, Bassi et al n'observaient pas d'association entre Ac anti-PTX3 et activité du LES [36]. Dans ce travail, les patients avec néphrite lupique avaient une prévalence moins importante d'Ac anti-PTX3. Toutefois, Bassi et al n'utilisaient pas le SLEDAI comme score d'évaluation d'activité du LES et l'étude ne comportait pas de suivi longitudinal des patients, limitant ainsi l'interprétation des résultats.

Finalement, les observations concernant les Ac anti-PTX3 au cours du LES sont très similaires de celles observées avec les Ac anti-CRP. Dans notre travail, nous avons pu étudier la spécificité des Ac anti-PTX3 détectés en ELISA. Deux approches ont été utilisées pour vérifier la spécificité des Ac détectés [4]. La première a consisté en une déplétion des Ac anti-CRP et anti-SAP de sérums double positifs en Ac anti-PTX3 et Ac anti-CRP ou anti-SAP. La déplétion des Ac anti-CRP ou SAP de ces sérums ne modifiait pas la détection des Ac anti-PTX3. Dans une seconde approche nous avons développé un ELISA pour détecter les Ac dirigés contre le domaine $\mathrm{N}$-terminal spécifique de PTX3, absent des PTXs courtes. Nous avons observé une corrélation très étroite du taux d'Ac anti-PTX3 dirigés contre le domaine C-terminal et du taux d'Ac anti-PTX3 dirigés contre le domaine $\mathrm{N}$-terminal.

\section{Anticorps anti-PTX3 au cours des vascularites à ANCA}

\section{Vascularites à ANCAs}

Les vascularites à ANCAs (VAA) sont des pathologies auto-immunes caractérisées par des atteintes inflammatoires et nécrosantes des vaisseaux de petit et moyen calibres [37, 38]. Ces entités, regroupant la granulomatose avec polyangéite (GPA, maladie de Wegener), la polyangéite microscopique (MPA) et la granulomatose hyperéosinophilique avec polyangéite (EGPA, maladie de Churg et Strauss), sont caractérisées par la présence d'auto-Ac dirigés contre des protéines stockées dans le cytoplasme des PNN, les ANCAs [37]. Toutefois, certains patients n'ont pas d'ANCA détectables avec les techniques de routine, mais il a pu être démontré qu'une partie de ces patients présentent réellement des ANCAs lorsque des techniques de détection plus élaborées sont utilisées [39]. Les ANCAs détectés au cours des VAA sont dirigés contre 2 cibles considérées comme les antigènes majeurs de ces pathologies: la myélopéroxydase (MPO) et la proteinase-3 (PR3). Les ANCA anti-PR3 donnent une fluorescence cytoplasmique en immunofluorescence indirecte (IFI) sur des PNN fixés et sont fortement associés à la GPA, alors que les ANCA antiMPO donnent une fluorescence péri-nucléaire et sont associés avec la MPA et l'EGPA [37].

En pratique clinique, après exclusion des principaux diagnostics différentiels (notamment les infections), l'association d'une présentation clinique compatible et la mise en évidence d'ANCA anti-MPO ou anti-PR3 sont souvent suffisantes pour initier un traitement immuno- 

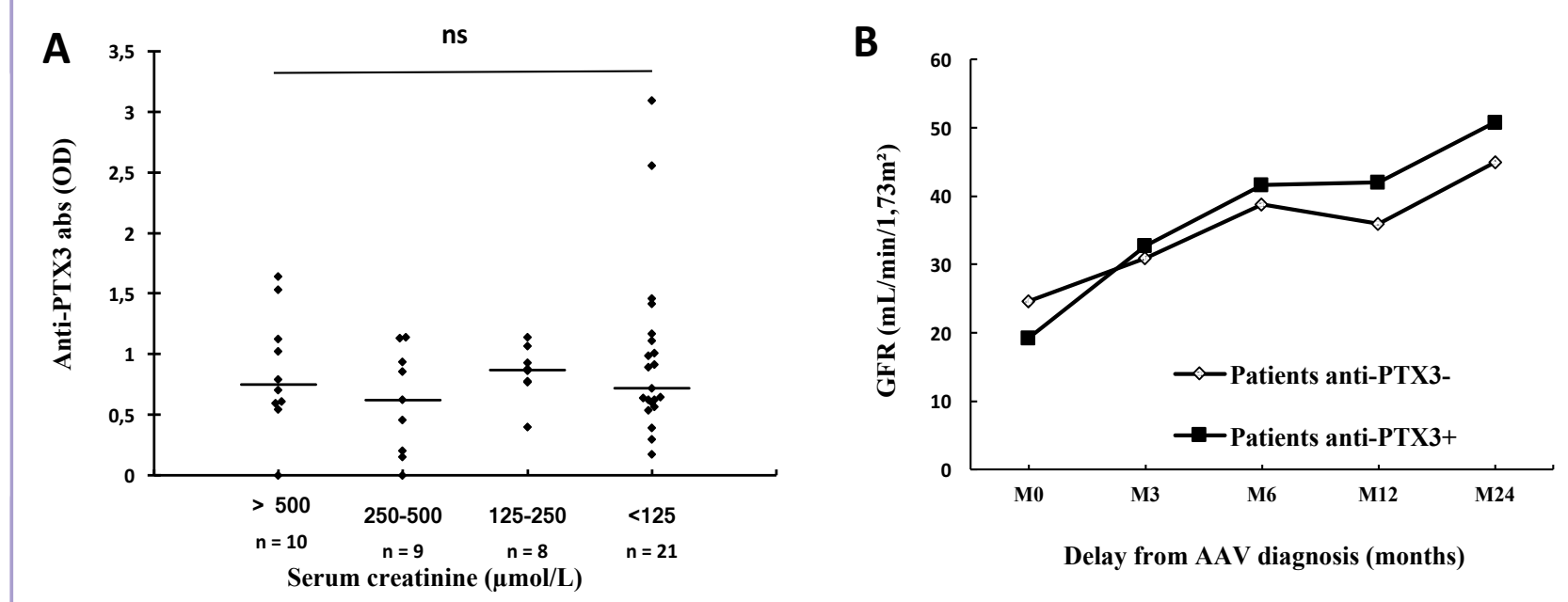

Les Ac anti-PTX3 ont été dosés par ELISA chez 48 patients ayant une atteinte rénale de VAA. A, Taux d'Ac anti-PTX3 selon la créatininémie au diagnostic. Le test de Mann-Withney a été utilisé pour les comparaisons de groupes. B, Débit de filtration glomérulaire depuis le diagnostic de VAA jusqu'à 24 mois de suivi en fonction de la présence ou non d'Ac anti-PTX3 au diagnostic.

suppresseur, particulièrement puissant en cas d'atteinte d'organe sévère (rénale, pulmonaire, cardiaque, neurologique) [40]. L'examen anatomo-pathologique d'un tissu atteint montrant des lésions de vascularite nécrosante des petits vaisseaux, permet de confirmer le diagnostic, mais n'est pas souvent réalisé immédiatement.

Toutefois, 10 à $15 \%$ des patients atteints de GPA ou de MPA, et jusqu'à $60 \%$ des patients atteints d'EGPA ne présentent pas d'ANCA détectables par les techniques de routine au cours de l'évolution de leur VAA [38]. Dans ces présentations, qualifiées de VAA ANCAnégatives, le diagnostic et l'initiation des thérapeutiques spécifiques se fait plus tardivement. Ces formes deVAA ANCA-négatives sont donc plus difficiles à diagnostiquer et sont associées à un plus mauvais pronostic fonctionnel et vital [39].

En plus des ANCAs dirigés contre des antigènes majeurs (MPO et PR3), des ANCAs dirigés contre des cibles antigéniques du PNN dites mineures telles que l'élastase, la lactoferrine, la BPI (bactericidal permeability increasing protein) ou la cathepsine $G$ peuvent être détectés au cours des VAA, mais aussi dans d'autres pathologies telles que les connectivites ou en contexte infectieux. Ces ANCAs dirigés contre des cibles mineures donnent le plus souvent un aspect de fluorescence atypique en IFI (a-ANCA). Leur signification au cours des VAA n'est pas déterminée [37].

Contrairement aux PTXs courtes produites essentiellement dans le foie, PTX3 est exprimée dans de nombreux tissus et par de nombreux types cellulaires. Les travaux menés au laboratoire ont montré que PTX3 était exprimée fortement dans les polynucléaires neutrophiles. PTX3 est stockée principalement dans les granules spécifiques du PNN, lactoferrine positives
[12]. Elle est libérée dans le milieu extracellulaire après activation du PNN par des stimuli pro-inflammatoires. Étant donné la rupture de tolérance vis-à-vis de différentes protéines stockées dans les granules du PNN au cours des VAA, nous avons émis l'hypothèse que PTX3 était également immunogène au cours des VAA.

\section{Anticorps anti-PTX 3 au cours des VAA}

Nous avons dosé les Ac anti-PTX3 dans les sérums de I 50 patients atteint de VAA ANCA positives et négatives [5]. Dans cette cohorte de patients, $79.7 \%$ et $85,1 \%$ des patients atteints de MPA et de GPA, respectivement, avaient des Ac anti-MPO ou anti-PR3, contre seulement $13,6 \%$ des patients atteint d'EGPA. Nous avons observé que $40 \%$ des patients présentaient des Ac anti-PTX3 au cours de l'évolution de leur maladie. Les Ac anti-PTX3 étaient plus fréquemment détectés chez les patients avec une maladie active $(45,2 \%$ contre $24,6 \%)$. Chez 11 patients, des sérums étaient disponibles au diagnostic de la vascularite en poussée, puis à distance après obtention de la rémission. Nous avons observé une diminution significative du titre des Ac anti-PTX3, passant au-dessous du seuil de positivité, après obtention de la rémission. Les Ac anti-PTX3 étaient associés à la présence concomitante d'ANCA anti-MPO ou d'ANCA anti-PR3 dans 36 et $35,1 \%$ des cas, respectivement.

Nous n'avons pas observé d'association des anticorps anti-PTX3 avec les atteintes cliniques au diagnostic de la VAA. Les patients avec atteinte rénale ont fait l'objet d'une étude approfondie. Nous n'avons pas observé de différence significative pour ce qui concerne la sévérité initiale et l'évolution de l'atteinte rénale entre les patients Ac anti-PTX3 positifs et négatives (figure 3 ). 


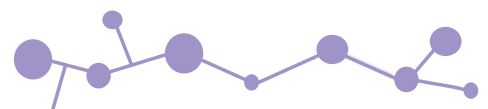

\section{Dossier scientifique}

\section{Figure 4. Aspect en immunofluorescence indirecte des Ac anti-PTX3}
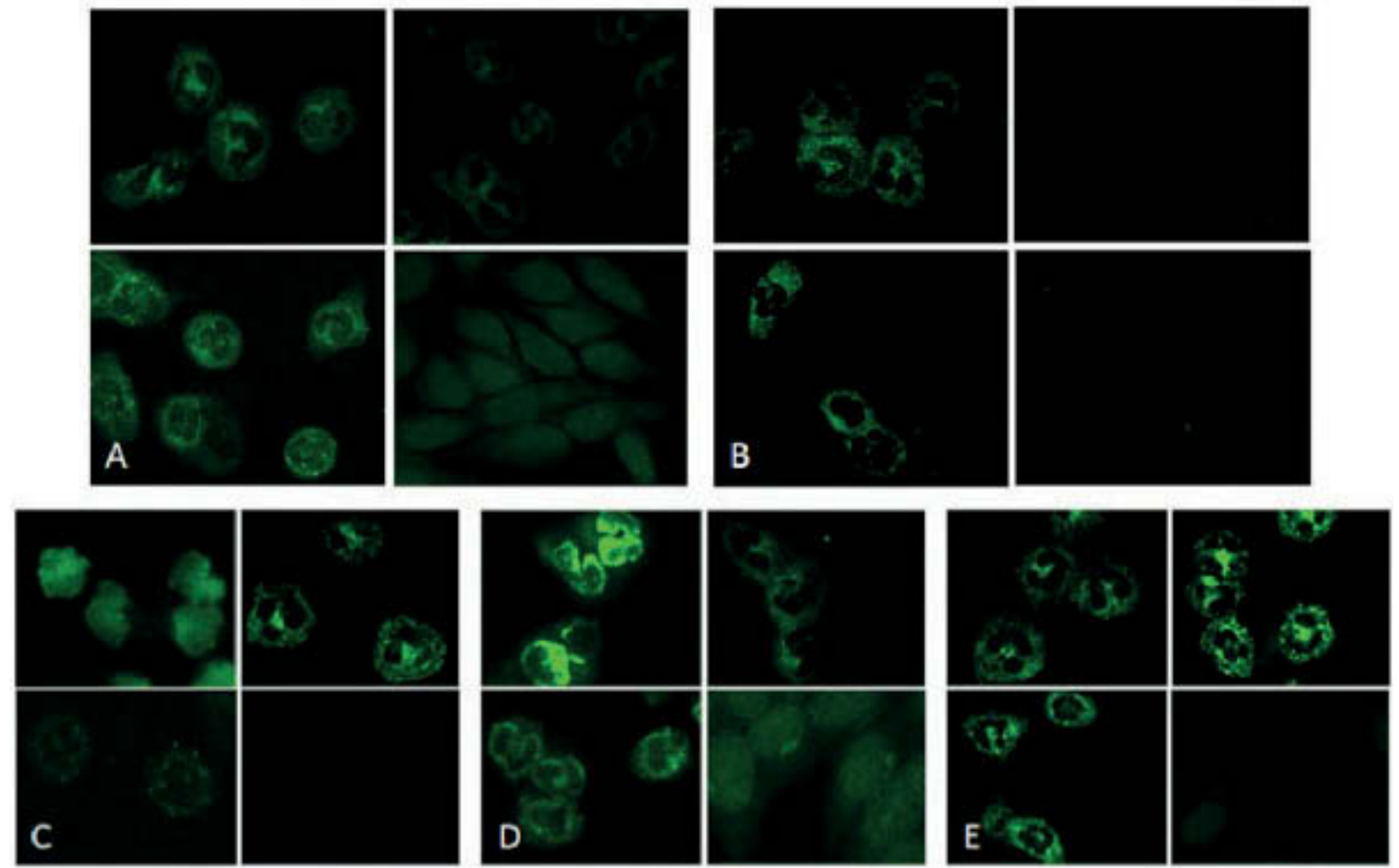

Aspect en immunofluorescence indirecte des Ac anti-PTX3 sur lame de polynucléaires fixés en éthanol (en haut à gauche), en formol (en haut à droite), en méthanol (en bas à gauche) et sur lame de cellules HEp2 (en bas à droite). $\mathbf{A}$, aspect de fluorescence obtenu avec le sérum d'un patient contenant des Ac anti-PTX3, mais ne contenant pas d'Ac anti-MPO, -PR3, -BPI, -lactoferrine, -elastase et -cathepsine G. B, aspect de fluorescence sur lame obtenu avec un Ac anti-PTX3 polyclonal de lapin. C-E, aspects typiques p-, a- et c-ANCA obtenus avec des sérums humains. Les photographies ont été prises par le Docteur Gilles Renier au Laboratoire d'Immunologie et d'Allergologie du CHU d'Angers.

\section{Anticorps anti-PTX3 au cours des vascularites MPO/PR3 négatives}

Nous avons étudié plus particulièrement les $A c$ antiPTX3 chez les patients atteints de VAA PR3 et MPO négatives. Dans notre cohorte, 14 patients (6 EGPA, 6 MPA et 2 GPA) n'avaient ni ANCA anti-MPO et ni ANCA anti-PR3 au diagnostic. De plus la recherche d'ANCA atypiques (par ELISA) dirigés contre les antigènes mineurs était également négative chez ces patients (lactoferrine, BPI, elastase, cathepsine G). Chez la moitié d'entre eux, des Ac anti-PTX3 étaient détectés au diagnostic de la vascularite. Ainsi, ces résultats suggèrent que les $A c$ anti-PTX 3 pourraient avoir un intérêt diagnostique au cours des vascularites ANCA négatives. II serait probablement très intéressant d'étudier la prévalence des Ac anti-PTX3 dans une plus importante population de patients atteints deVAAANCA négatives afin de préciser leur intérêt clinique.
Les Ac anti-PTX3 sont-ils des ANCAs?

Étant donné la forte prévalence des Ac anti-PTX3 au cours des VAA et que PTX 3 est stockée dans les granules spécifiques des PNN [12], nous avons émis l'hypothèse que les Ac anti-PTX3 étaient des ANCA. Différentes caractéristiques doivent être vérifiées afin d'affirmer qu'un Ac est un ANCA: la cible antigénique doit être une molécule présente dans le cytoplasme du PNN, une positivité de l'IFI sur PNN fixés sur lame doit être observée, et enfin, l'Ac doit pouvoir induire une activation du PNN. Les Ac anti-PTX3 remplissent le premier critère.

Nous avons mené les expérimentations suivantes pour étudier le second critère. Nous avons étudié les sérums de 7 patients présentant des Ac anti-PTX3, sans réactivité vis-à-vis des autres cibles antigéniques des ANCA (MPO, PR3, BPI, lactoferrine, cathepsine G). L'aspect de fluorescence en IFI sur PNN fixés en éthanol, 


\section{scientifique Immunologie}

formol-acétone et en méthanol était étudié. Sur les 7 sérums étudiés, 4 avaient une IFI positive. Ces 4 sérums étaient responsables d'un aspect de fluorescence tout à fait caractéristique et concordant, similaire à celui obtenu en utilisant un Ac de lapin dirigé contre la PTX3 humaine (contrôle positif). En éthanol, l'aspect de fluorescence était différent de l'aspect $p$ - et a-ANCA, mais assez proche de l'aspect c-ANCA.Toutefois, les granules cytoplasmiques apparaissaient plus petits, bien visibles en éthanol et méthanol, mais moins détectables en formol. Nous avons nommé cet aspect «small cytoplasmic » ANCA (sc-ANCA) (figure 4). Le troisième critère correspondant à l'action biologique des Ac anti-PTX3 n'a pas encore été étudié. Pour cela, une purification des Ac anti-PTX3 à partir du sérum humain sur colonne d'affinité couplée à l'antigène (PTX3) ou une purification des immunoglobulines d'un patient ayant exclusivement des Ac anti-PTX3 (sans autre ANCA) serait nécessaire. L'action biologique des Ac anti-PTX3 pourrait alors être étudiée in vitro.

\section{Conclusion}

Les données disponibles concernant les Ac anti-PTX3 suggèrent que ces $A c$ pourraient être des marqueurs d'intérêt pour le diagnostic et le suivi, autant du lupus systémique que des vascularites à ANCA.Toutefois, des études prospectives avec un suivi longitudinal des patients sont nécessaires pour caractériser de façon précise l'apport de ce marqueur comparativement à ceux déjà utilisés en pratique clinique. Étant donné l'absence de marqueur disponible au cours des VAAANCA négatives, l'intérêt des Ac anti-PTX3 dans cette situation précise pourrait être majeur.

Liens d'intérêts: les auteurs déclarent ne pas avoir de liens d'intérêts.

\section{Points à retenir}

- Les pentraxines sont des PRRs solubles impliqués au cours de la défense antimicrobienne et l'efferocytose.

- Il existe une rupture de tolérance vis-à-vis des pentraxines aux cours de différentes maladies autoimmunes.

- Des auto-anticorps anti-PTX3 sont détectés à prévalence élevée au cours du lupus systémique et des vascularites à ANCA et pourraient avoir un intérêt pour l'évaluation de l'activité de ces maladies.

- Les Ac anti-PTX3 partagent certaines caractéristiques des ANCAs et sont associés à un aspect de fluorescence spécifique en IFI sur polynucléaire neutrophile.

- La prévalence des Ac anti-PTX3 semble élevée au cours des VAA ANCA négatives et pourrait en constituer un marqueur d'utilité clinique.

\section{Références}

[I] Jeannin P, Jaillon S, Delneste Y. Pattern recognition receptors in the immune response against dying cells. Current opinion in immunology 2008, 20(5):530-7.

[2] Kawasaki T, Kawai T, Akira S. Recognition of nucleic acids by patternrecognition receptors and its relevance in autoimmunity. Immunological reviews 2011, 243(1):61-73.

[3] Ortega-Hernandez OD, Bassi N, Shoenfeld Y, et al. The long pentraxin 3 and its role in autoimmunity. Seminars in arthritis and rheumatism 2009, 39(1):38-54.

[4] Augusto JF, Onno C, Blanchard S, et al. Detection of anti-PTX3 autoantibodies in systemic lupus erythematosus. Rheumatology 2009, 48(4):442-4.

[5] Simon A, Subra JF, Guilpain P, et al. Detection of Anti-Pentraxin-3 Autoantibodies in ANCA-Associated Vasculitis. PloS one 2016, 11(1):e0147091.

[6] Deban L, Jaillon S, Garlanda C, et al. Pentraxins in innate immunity: lessons from PTX3. Cell and tissue research 2011, 343(1):237-49.

[7] Szalai AJ, van Ginkel FW, Wang Y, et al. Complement-dependent acute-phase expression of C-reactive protein and serum amyloid P-component. Journal of immunology 2000, 165(2):1030-5.

[8] Garlanda C, Bottazzi B, Bastone A, et al. Pentraxins at the crossroads between innate immunity, inflammation, matrix deposition, and female fertility. Annual review of immunology 2005, 23:337-66. [9] Pepys MB, Hirschfield GM. C-reactive protein: a critical update. The Journal of clinical investigation 2003, 111(12):1805-12.

[I 0] Latini R, Maggioni AP, Peri G, et al. Prognostic significance of the long pentraxin PTX3 in acute myocardial infarction. Circulation 2004 110(16):2349-54.
[I I] Muller B, Peri G, Doni A, et al. Circulating levels of the long pentraxin PTX3 correlate with severity of infection in critically ill patients. Critical care medicine 2001, 29(7):1404-7.

[I 2] Jaillon S, Peri G, Delneste Y, et al. The humoral pattern recognition receptor PTX3 is stored in neutrophil granules and localizes in extracellular traps. The Journal of experimental medicine 2007, 204(4):793-804. [13] Kessenbrock K, Krumbholz M, Schonermarck U, et al. Netting neutrophils in autoimmune small-vessel vasculitis. Nature medicine 2009, 15(6):623-5.

[I 4] Mold C, Rodgers CP, Kaplan RL, et al. Binding of human C-reactive protein to bacteria. Infection and immunity 1982, 38(1):392-5.

[15] Weiser JN, Pan N, McGowan KL, et al. Phosphorylcholine on the lipopolysaccharide of Haemophilus influenzae contributes to persistence in the respiratory tract and sensitivity to serum killing mediated by C-reactive protein. The Journal of experimental medicine 1998, 187(4):631-40.

[16] Noursadeghi M, Bickerstaff MC, Gallimore JR, et al. Role of serum amyloid $\mathrm{P}$ component in bacterial infection: protection of the host or protection of the pathogen. Proceedings of the National Academy of Sciences of the United States of America 2000, 97(26):14584-9. [ 17 ] Szalai AJ, VanCott JL, McGhee JR, et al. Human C-reactive protein is protective against fatal Salmonella enterica serovar typhimurium infection in transgenic mice. Infection and immunity 2000, 68(10):5652-6. [18] Lech M, Rommele C, Kulkarni OP, et al. Lack of the long pentraxin PTX3 promotes autoimmune lung disease but not glomerulonephritis in murine systemic lupus erythematosus. PloS one 2011, 6(5):e20118. [1 9] Szondy Z, Garabuczi E, Joos G, et al. Impaired clearance of apoptotic cells in chronic inflammatory diseases: therapeutic implications. Frontiers in immunology 2014, 5:354. 
[20] Mihlan M, Stippa S, Jozsi M, et al. Monomeric CRP contributes to complement control in fluid phase and on cellular surfaces and increases phagocytosis by recruiting factor $\mathrm{H}$. Cell death and differentiation 2009, 16(12):1630-40.

[2I] van Rossum AP, Fazzini F, Limburg PC, et al. The prototypic tissue pentraxin PTX3, in contrast to the short pentraxin serum amyloid P, inhibits phagocytosis of late apoptotic neutrophils by macrophages. Arthritis and rheumatism 2004, 50(8):2667-74.

[22] Rodriguez W, Mold C, Marnell LL, et al. Prevention and reversal of nephritis in MRL/lpr mice with a single injection of C-reactive protein. Arthritis and rheumatism 2006, 54(1):325-35.

[23] Szalai AJ. C-reactive protein (CRP) and autoimmune disease: facts and conjectures. Clinical \& developmental immunology 2004, 11(34):221-6.

[24] Gillmore JD, Hutchinson WL, Herbert J, et al. Autoimmunity and glomerulonephritis in mice with targeted deletion of the serum amyloid $\mathrm{P}$ component gene: SAP deficiency or strain combination? Immunology 2004, 112(2):255-64.

[25] Firooz N, Albert DA, Wallace DJ, et al. High-sensitivity C-reactive protein and erythrocyte sedimentation rate in systemic lupus erythematosus. Lupus 2011, 20(6):588-97.

[26] Robey FA, Jones KD, Steinberg AD. C-reactive protein mediates the solubilization of nuclear DNA by complement in vitro. The Journal of experimental medicine 1985, 161(6):1344-56.

[27] Bell SA, Faust $\mathrm{H}$, Schmid A, et al. Autoantibodies to C-reactive protein (CRP) and other acute-phase proteins in systemic autoimmune diseases. Clinical and experimental immunology 1998, 113(3):327-32.

[28] Eisenhardt SU, Thiele JR, Bannasch H, et al. C-reactive protein: how conformational changes influence inflammatory properties. Cell cycle 2009, 8(23):3885-92.

[29] Shoenfeld Y, Szyper-Kravitz M, Witte T, et al. Autoantibodies against protective molecules--C1q, C-reactive protein, serum amyloid $\mathrm{P}$, mannose-binding lectin, and apolipoprotein A1: prevalence in systemic lupus erythematosus. Annals of the New York Academy of Sciences 2007, 1108:227-39.

[30] Sjowall C, Bengtsson AA, Sturfelt G, et al. Anti-CRP autoantibody levels correlate with disease activity in systemic lupus erythe- matosus. Seminars in arthritis and rheumatism 2005, 35(1):65; author reply 66.

[3 I] Figueredo MA, Rodriguez A, Ruiz-Yague M, et al. Autoantibodies against C-reactive protein: clinical associations in systemic lupus erythematosus and primary antiphospholipid syndrome. The Journal of rheumatology 2006, 33(10):1980-6.

[32] Sjowall C, Zickert A, Skogh T, et al. Serum levels of autoantibodies against $\mathrm{C}$-reactive protein correlate with renal disease activity and response to therapy in lupus nephritis. Arthritis research \& therapy 2009, 11(6):R188.

[33] Tan $\mathrm{Y}, \mathrm{Yu} F$, Yang $\mathrm{H}$, et al. Autoantibodies against monomeric C-reactive protein in sera from patients with lupus nephritis are associated with disease activity and renal tubulointerstitial lesions. Human immunology 2008, 69(12):840-4.

[34] Tan Y, Yu F, Qu Z, et al. Modified C-reactive protein might be a target autoantigen of TINU syndrome. Clinical journal of the American Society of Nephrology: CJASN 2011, 6(1):93-100.

[35] Zandman-Goddard G, Blank M, Langevitz P, et al. Anti-serum amyloid component $P$ antibodies in patients with systemic lupus erythematosus correlate with disease activity. Annals of the rheumatic diseases 2005, 64(12):1698-702.

[36] Bassi N, Ghirardello A, Blank M, et al. IgG anti-pentraxin 3 antibodies in systemic lupus erythematosus. Annals of the rheumatic diseases 2010, 69(9):1704-10.

[37] Beauvillain C, Delneste Y, Renier G, et al. Antineutrophil cytoplasmic autoantibodies: how should the biologist manage them? Clinical reviews in allergy \& immunology 2008, 35(1-2):47-58.

[38] Jennette JC, Falk RJ, Hu P, et al. Pathogenesis of antineutrophil cytoplasmic autoantibody-associated small-vessel vasculitis. Annual review of pathology 2013, 8:139-60.

[39] Roth AJ, Ooi JD, Hess JJ, et al. Epitope specificity determines pathogenicity and detectability in ANCA-associated vasculitis. The Journal of clinical investigation 2013, 123(4):1773-83.

[40] Sayegh J, Poli C, Chevailler A, et al. Emergency testing for antineutrophil cytoplasmic antibodies combined with a dialog-based policy between clinician and biologist: effectiveness for the diagnosis of ANCAassociated vasculitis. Internal and emergency medicine 2014. 